\title{
IEDITORIAL
}

\section{Balloon aortic valvuloplasty in the contemporary era}

\author{
Hassan Kamran', Deepthi Sudhakar'1, Hani Jneid ${ }^{1,2}$ \\ 1 Division of Cardiology, Baylor College of Medicine, Houston, Texas, United States \\ 2 Division of Cardiology, Michael E. DeBakey Veterans Affairs Medical Center, Houston, Texas, United States
}

\section{RELATED ARTICLE} by Tyczyński et al, see p. 982

Correspondence to: Hani Jneid, MD, Division of Cardiology, Baylor School of Medicine and the Michael E. DeBakey Veterans Affairs Medical Center, 2002 Holcombe Blvd., VAMC-VA111, 77030 Houston, Texas, United States, phone: +1 (713) 7947300 , email:jneid@bcm.edu Received: October 7, 2020. Accepted: October 8, 2020. Published online: October 23, 2020. Kardiol Pol. 2020; 78 (10): 956-958 doi:10.33963/KP.15652

Copyright by the Author(s), 2020
Balloon aortic valvuloplasty (BAV) was first introduced by Alain Cribier in 1986 as a therapeutic option for nonoperative patients with severe aortic stenosis (AS). ${ }^{1}$ While immediate hemodynamic parameters did improve with BAV, these improvements were modest at best, ameliorating the aortic valve gradient from severe to the less severe or moderate-to-severe range, and were only sustained for few weeks or months after the BAV procedure. In addition, the high mortality and complications associated with the early BAV procedure and its limited impact on long-term survival further decreased the enthusiasm for this procedure and limited its use to a palliative approach. ${ }^{2}$ The introduction of transcatheter aortic valve replacement (TAVR), however, has renewed interest in BAV as a bridge to definitive treatment or to decision making. From 2004 to 2013, the annual number of BAV cases increased from 707 to 3715 in the United States. ${ }^{3}$ The 2014 American Heart Association / American College of Cardiology guidelines for the management of valvular heart disease provided a class IIb recommendation for BAV stating that it may be considered a bridge to surgical or transcatheter aortic valve replacement in patients with severe symptomatic AS. ${ }^{4}$ The 2017 European Society of Cardiology guidelines provided several class IIb indications for BAV in patients with severe AS, including for palliative purposes, in those requiring urgent noncardiac surgery, for diagnostic purposes in patients with other confounding symptoms, and in those with other reversible organ dysfunctions to assess the response to BAV and potential benefit from subsequent escalation to TAVR. 5

Balloon aortic valvuloplasty is therefore an important procedure that should remain in the armamentarium of all interventional cardiologists, especially those performing structural heart disease interventions. It is in this context that the study by Tyczyński et $\mathrm{al}^{6}$ should be viewed. The investigators performed a retrospective analysis identifying patients who underwent elective and rescue BAV from July 2010 to August 2018 in their tertiary care center. Of the 35 cases identified, 16 were elective and 19 were rescue BAV procedures. Elective BAV was performed either for diagnostic purposes in patients with other potential causes of symptoms or as a therapeutic strategy for hemodynamically stable patients with severe myocardial dysfunction who had contraindications to TAVR. Rescue BAV was undertaken in patients with pulmonary edema, prior resuscitation, or cardiogenic shock requiring intravenous inotropes and diuretics. Of note, a successful BAV procedure, defined as $\geq 50 \%$ reduction in the mean aortic gradient without resultant moderate or severe aortic insufficiency, was only achieved in a single patient in the elective group. The reduction in the mean aortic pressure gradient was $5 \mathrm{~mm} \mathrm{Hg}$ on average. In the rescue-BAV group, the periprocedural mortality rate was $21 \%$ (4 patients) compared with $0 \%$ in the elective group $(P=0.1)$. In-hospital mortality was notably higher in the rescue group at $63.1 \%$ (12 patients) compared with the elective group (3 patients [18.7\%]; $P<0.01)$. The authors appropriately noted that the mortality observed in their study was higher than that presented in previously published reports and attributed it to a sicker patient population. They concluded that in-hospital mortality after BAV remained high and identified higher EuroSCORE II, intravenous diuretic use, intravenous inotrope use, and urgency of the procedure as predictors of increased mortality. 
A successful BAV procedure depends on multiple factors including the severity of the gradient and the calcification of the valve itself, with higher gradients and more marked calcification portending less success. Success of BAV in congenital AS, as defined by post catheter-measured peak systolic ejection gradient $<35 \mathrm{~mm} \mathrm{Hg}$ and mild or not worse than baseline aortic regurgitation, has been achieved in up to $70.9 \%$ of patients. ${ }^{7}$ However, the pathology of calcific degenerative AS in older patients is completely different and involves significant leaflet calcification, thickening and mobility restriction, often with annular involvement, and aortic calcification. Senile calcific AS is not a predominantly commissural disease, as seen for example in rheumatic aortic valve disease where BAV is a definitive therapy. We conducted an earlier systematic review encompassing 27 studies and 4123 adult patients undergoing BAV for severe AS and demonstrated a change in the mean aortic gradient from $50.3 \mathrm{~mm} \mathrm{Hg}$ to $24.6 \mathrm{~mm} \mathrm{Hg}$, an overall procedural mortality of $2.2 \%$, and in-hospital mortality of $7.1 \%$ with BAV. ${ }^{8}$ We also shown an improvement in outcomes in the contemporary era (after 2005) compared with the pre-TAVR era, with decreased procedural and in-hospital mortality $(1.5 \%$ vs $2.9 \%$ and $4.6 \%$ vs $8.5 \%$, respectively) despite patients having more comorbidities (including a higher prevalence of heart failure) in the contemporary era. This may be attributed to increased operators' experience with BAV and to technical and procedural improvements, including the use of a single-balloon inflation, rapid pacing, and the retrograde approach. Notably, we have shown that BAV using an anterograde approach may provide similar long-term outcomes to the retrograde approach and is associated with lesser vascular complications. ${ }^{9}$ However, the antegrade BAV approach has been largely abandoned, because it involves a trans-septal access, is generally more complex, and requires a steeper learning curve and advanced expertise. An antegrade BAV approach may be reserved to selected patients with severe $A S$ and significant peripheral arterial disease in whom a retrograde approach is not feasible.

The high mortality associated with the rescue BAV procedure in the study by Tyczyński et $\mathrm{al}^{6}$ is not surprising. Predictors of in-hospital death after BAV include cardiogenic shock, coagulopathy, the need for mechanical support, and low institutional volume of BAV procedures. ${ }^{3}$ In a study of 44 patients presenting with cardiogenic shock due to acutely decompensated severe AS who underwent BAV, the rate of 1-month mortality was $47 \% .^{10}$ Univariate predictors of mortality included the preoperative use of dobutamine $>5 \mu \mathrm{g} / \mathrm{kg} / \mathrm{min}$ and delayed BAV performed later than 48 hours after presentation. One-year mortality or recurrence of cardiogenic shock was found to be $90 \%$ if BAV was delayed versus $59 \%$ if it was performed within the first 48 hours. However, these patients had a mean EuroSCORE II of $41.6 \%$, compared with the average EuroSCORE II of $25 \%$ in the current study by Tyczyński et al. ${ }^{6}$ Overall, a substantial mortality has been observed in patients with cardiogenic shock and severe AS who need urgent BAV. Advanced mechanical support (such as a tandem heart when available) and an expeditious BAV procedure undertaken before profound hypoperfusion, severe multiorgan dysfunction, and metabolic derangements occur may be needed to reduce mortality in these patients. Studies have also shown that a low institutional volume of BAV is associated with worse outcomes, with centers at the lowest quartile of annual procedure volume (1 to 2 procedures per year) having a 1.58-fold increased risk of in-hospital mortality compared with the highest volume centers ( $\geq 18$ procedures per year). ${ }^{3}$ It is therefore important for elective $\mathrm{BAV}$ procedures to be performed in high-volume centers and for their appropriateness and outcomes to be evaluated, monitored, and benchmarked against similar hospitals and the reported literature.

The study by Tyczyński et $\mathrm{al}^{6}$ adds to the current literature on the contemporary outcomes of BAV with a direct comparison of elective versus urgent BAV. Not unexpectedly, they demonstrated higher mortality in patients presenting for urgent BAV compared with their elective counterparts. With the advent of TAVR, there has been an increase in the adoption of BAV as a bridge to definitive treatment. However, given the risks associated with subjecting the patient to 2 procedures in a stepwise approach (BAV followed by staged TAVR), many experts are now advocating to proceed with TAVR directly, especially given the technical advancement and increased expertise with TAVR. This study in a way reinforces this notion: although BAV outcomes have improved in the contemporary era due to increased operators' experience and procedural refinement, the mortality in the setting of rescue BAV remains unacceptably high.

\section{ARTICLE INFORMATION}

DISCLAIMER The opinions expressed by the author are not necessarily those of the journal editors, Polish Cardiac Society, or publisher.

\section{CONFLICT OF INTEREST None declared.}

OPEN ACCESS This is an Open Access article distributed under the terms of the Creative Commons Attribution-NonCommercial-NoDerivatives 4.0 International License (CC BY-NC-ND 4.0), allowing third parties to download articles and share them with others, provided the original work is properly cited, not changed in any way, distributed under the same license, and used for noncommercial purposes only. For commercial use, please contact the journal office at kardiologiapolska@ptkardio.pl.

HOW TO CITE Kamran H, Sudhakar D, Jneid H. Balloon aortic valvuloplasty in the contemporary era. Kardiol Pol. 2020; 78: 956-958. doi:10.33963/KP.15652

\section{REFERENCES}

1 Cribier A, Savin T, Saoudi N, et al. Percutaneous transluminal aortic valvuloplasty using a balloon catheter. A new therapeutic option in aortic stenosis in the elderly. Arch Mal Coeur Vaiss. 1986; 79: 1678-1686.

2 Lieberman EB, Bashore TM, Hermiller JB, et al. Balloon aortic valvuloplasty in adults: failure of procedure to improve long-term survival. J Am Coll Cardiol. 1995; 26: 1522-1528. 
3 Alkhouli M, Zack CJ, Sarraf M, et al. Morbidity and mortality associated with balloon aortic valvuloplasty: a national perspective. Circ Cardiovasc Interv. 2017; 10: e004481.

4 Nishimura RA, Otto CM, Bonow RO, et al. 2017 AHA/ACC focused update of the $2014 \mathrm{AHA} / \mathrm{ACC}$ guideline for the management of patients with valvular heart disease. J Am Coll Cardiol. 2017; 135: e1159-e1195.

5 Baumgartner H, Falk V, Bax JJ, et al. 2017 ESC/EACTS Guidelines for the management of valvular heart disease. Eur Heart J. 2017; 38: 2739-2791.

6 Tyczyński P, Chmielak Z, Dąbrowski M, et al. Elective versus rescue balloon aortic valvuloplasty for critical aortic stenosis. Kardiol Pol. 2020; 78: 982-989.

7 Boe BA, Zampi JD, Kennedy KF, et al. Acute success of balloon aortic valvuloplasty in the current era: a national cardiovascular data registry study. JACC Cardiovasc Interv. 2017; 10: 1717-1726.

8 Kumar A, Paniagua D, Hira RS, et al. Balloon aortic valvuloplasty in the transcatheter aortic valve replacement era. Invasive Cardiol. 2016; 28: 341-348.

9 Cubeddu RJ, Jneid H, Don CW, et al. Retrograde versus antegrade percutaneous aortic balloon valvuloplasty: Immediate, short- and long-term outcome at 2 years. Catheter Cardiovasc Interv. 2009: 74: 225-231.

10 Debry $\mathrm{N}$, Kone $\mathrm{P}$, Vincent $\mathrm{F}$, et al. Urgent balloon aortic valvuloplasty in patients with cardiogenic shock related to severe aortic stenosis: time matters. EuroIntervention. 2018; 14: e519-e525. 